\title{
Concavity and Efficient Points of Discrete Distributions in Probabilistic Programming
}

\author{
Darinka Dentcheva András Prékopa \\ Andrzej Ruszczyński \\ RUTCOR, Rutgers Center for Operations Research \\ Rutgers University, Piscataway, NJ 08854, U.S.A.
}

\begin{abstract}
We consider stochastic programming problems with probabilistic constraints involving integer-valued random variables. The concept of a $p$-efficient point of a probability distribution is used to derive various equivalent problem formulations. Next we introduce the concept of $r$-concave discrete probability distributions and analyse its relevance for problems under consideration. These notions are used to derive lower and upper bounds for the optimal value of probabilistically constrained stochastic programming problems with discrete random variables. The results are illustrated with numerical examples.
\end{abstract}

\section{Introduction}

Probabilistic constraints are one of the main challenges of modern stochastic programming. Their motivation is clear: if in the linear program

$$
\begin{aligned}
\min & c^{T} x \\
\text { subject to } & T x \geq \xi \\
& A x \geq b \\
& x \geq 0
\end{aligned}
$$

the vector $\xi$ is random, we require that $T x \geq \xi$ shall hold at least with some prescribed probability $p \in(0,1)$, rather than for all possible realizations of the right hand side. This leads to the following problem formulation:

$$
\begin{aligned}
\min & c^{T} x \\
\text { subject to } & I P\{T x \geq \xi\} \geq p \\
& A x \geq b \\
& x \geq 0
\end{aligned}
$$

where the symbol $\mathbb{P}$ denotes probability. 
Programming under probabilistic constraints was initiated by Charnes, Cooper and Symonds in [7]. They formulated probabilistic constraints individually for each stochastic constraint. Joint probabilistic constraints for independent random variables were used first by Miller and Wagner in [16]. The general case was introduced and first studied by the second author of the present paper in [21, 24].

Much is known about problem (1.1) in the case where $\xi$ has a continuous probability distribution (see [26] and the references therein). However, only a few papers handle the case of a discrete distribution. In [25] a dual type algorithm for solving problem (1.1) has been proposed. Bounds for the optimal value of this problem, based on disjunctive programming, were analyzed in [32]. The case when the matrix $T$ is random, while $\xi$ is not, has been considered in [34]. Recently, in [27], a cutting plane method for solving (1.1) has been presented.

Even though the literature for handling probabilistic constraints with discrete random variables is scarce, the number of potential applications is large. Singh at al.in [33] consider a microelectronic wafer design problem that arises in semiconductor manufacturing. The problem was to maximize the probability rather than to optimize an objective function subject to a probabilistic constraint, but other formulations are possible as well. Another application area are communication and transportation network capacity expansion problems, where arc and node capacities are restricted to be integers [20, 26]. Bond portfolio problems with random integer-valued liabilities can be formalized as (1.1) (see [9] for first such attempts). Many production planning problems involving random indivisible demands fit to our general setting as well.

If the decision vector $x$ in (1.1) is restricted to be integer and $T$ is integer, then there is no need to consider other right hand side vectors than integer. In fact, for any random vector $\eta$ we have then $\mathbb{P}\{T x \geq \eta\}=\mathbb{P}\{T x \geq \xi\}$, where $\xi=\lceil\eta\rceil$ (the roundup of $\eta$ ). This transformation may additionally strengthen the description of the feasible set by deleting some non-integer points.

In section 2 we introduce the key concept of a $p$-efficient point of a discrete distribution and we analyse properties of such points. In section 3 we define the class of $r$-concave distribution functions of discrete random variables and we show how $r$-concavity can be used to derive various equivalent formulations of probabilistically constrained problems. Section 4 discusses a Lagrangian relaxation of the problem. In section 5 we propose a new method, called the cone generation method, for generating lower bounds of probabilistically constrained problems. Section 6 is devoted to upper bounds. Finally, in section 7 we present two illustrating examples.

Although we concentrate on integer random variables, all our results easily extend to other discrete distributions with non-uniform grids, under the condition that a uniform lower bound on the distance of grid points in each coordinate can be found.

To fix some notation we assume that in the problems above $A$ is an $m \times n$ matrix, $T$ is an $s \times n$ matrix; $c, x \in \mathbb{R}^{n}, b \in \mathbb{R}^{m}$ and $\xi$ is a random vector with values in $\mathbb{R}^{s}$. We use $\mathbb{Z}$ and $\mathbb{Z}_{+}$to denote the set of integers and nonnegative integers, respectively. The inequality ' $\geq$ ' for vectors is always understood coordinate-wise. 


\section{$2 p$-Efficient Points}

Let us define the sets:

$$
\mathcal{D}=\left\{x \in \mathbb{R}^{n}: A x \geq b, \quad x \geq 0\right\}
$$

and

$$
\mathcal{Z}_{p}=\left\{y \in \mathbb{R}^{s}: \mathbb{P}(\xi \leq y) \geq p\right\} .
$$

Clearly, problem (1.1) can be compactly rewritten as

$$
\begin{aligned}
\min & c^{T} x \\
\text { subject to } & T x \in \mathcal{Z}_{p} \\
& x \in \mathcal{D}
\end{aligned}
$$

While the set $\mathcal{D}$ is a convex polyhedron, the structure of $\mathcal{Z}_{p}$ needs to be analysed in more detail.

Let $F$ denote the probability distribution function of $\xi$, and $F_{i}$ the marginal probability distribution function of the $i$ th component $\xi_{i}$. By assumption, the set $\mathcal{Z}$ of all possible values of the random vector $\xi$ is included in $\mathbb{Z}^{s}$.

We shall use the concept of a $p$-efficient point, introduced in [25].

Definition 2.1. Let $p \in[0,1]$. A point $v \in \mathbb{R}^{s}$ is called a p-efficient point of the probability distribution function $F$, if $F(v) \geq p$ and there is no $y \leq v, y \neq v$ such that $F(y) \geq p$.

Obviously, for a scalar random variable $\xi$ and for every $p \in(0,1)$ there is exactly one $p$-efficient point: the smallest $v$ such that $F(v) \geq p$. Since $F(v) \leq F_{i}\left(v_{i}\right)$ for every $v \in \mathbb{R}^{s}$ and $i=1, \ldots, s$, we have the following result.

Lemma 2.2. Let $p \in(0,1)$ and let $l_{i}$ be the p-efficient point of the one-dimensional marginal distribution $F_{i}, i=1, \ldots, s$. Then every $v \in \mathbb{R}^{s}$ such that $F(v) \geq p$ must satisfy the inequality $v \geq l=\left(l_{1}, \ldots, l_{s}\right)$.

Rounding down to the nearest integer does not change the value of the distribution function, so $p$-efficient points of a random vector with all integer components (shortly, integer random vector) must be integer. We can thus use Lemma 2.2 to get the following interesting fact (noticed earlier in [35] for non-negative integer random variables).

Theorem 2.3. For each $p \in(0,1)$ the set of $p$-efficient points of an integer random vector is nonempty and finite.

Proof. The result follows from Dickson's Lemma [3, Cor. 4.48] and Lemma 2.2. For convenience we provide a short proof here.

We shall at first show that at least one $p$-efficient point exists. Since $p<1$, there must exist $y$ such that $F(y) \geq p$. By Lemma 2.2, all $v$ such that $F(v) \geq p$ are bounded below 


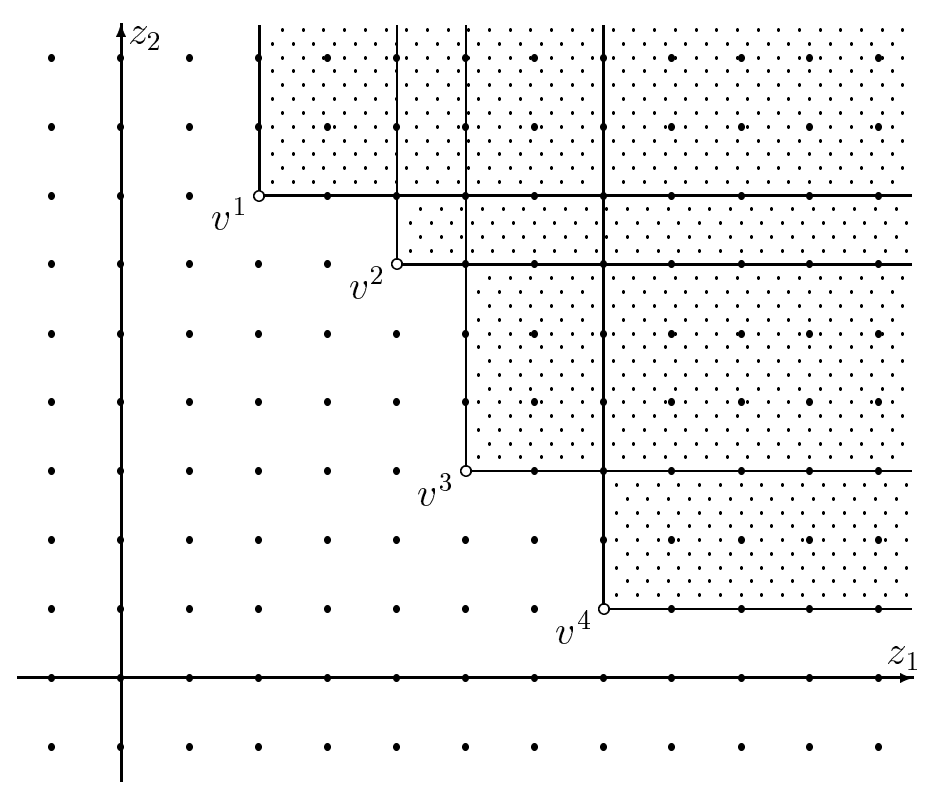

Figure 2.1: Example of the set $\mathcal{Z}_{p}$ with $p$-efficient points $v^{1}, \ldots, v^{4}$.

by the vector $l$ of $p$-efficient points of one-dimensional marginals. Therefore, if $y$ is not $p$-efficient, one of finitely many integer points $v$ such that $l \leq v \leq y$ must be $p$-efficient.

We shall now prove the finiteness of the set of $p$-efficient points. Suppose that there exisits an infinite sequence of different $p$-efficient points $v^{j}, j=1,2, \ldots$. Since they are integer, and the first coordinate $v_{1}^{j}$ is bounded from below by $l_{1}$, with no loss of generality we may select a subsequence which is non-decreasing in the first coordinate. By a similar token, we can select further subsequences which are non-decreasing in the first $k$ coordinates $(k=1, \ldots, s)$. Since the dimension $s$ is finite, we obtain a subsequence of different $p$-efficient points which is non-decreasing in all coordinates. This contradicts the definition of a $p$-efficient point.

Our proof can be easily adapted to the case of non-uniform grids for which a uniform lower bound on the distance of grid points in each coordinate exists.

Let $p \in(0,1)$ and let $v^{j}, j \in J$, be all $p$-efficient points of $\xi$. By Theorem 2.3, $J$ is a finite set. Let us define the cones

$$
K_{j}=v^{j}+\mathbb{R}_{+}^{s}, \quad j \in J
$$

Remark 2.4. $\mathcal{Z}_{p}=\bigcup_{j \in J} K_{j}$.

Proof. If $y \in \mathcal{Z}_{p}$ then either $y$ is $p$-efficient or there exists an integer $v \leq y, v \neq y$, $v \in \mathcal{Z}_{p}$. By Lemma 2.2, one must have $l \leq v$. Since there are only finitely many integer points $l \leq v \leq y$ one of them, $v_{j}$, must be $p$-efficient, and so $y \in K_{j}$.

Figure 2.1 illustrates this formulation. 
Thus, we obtain (for $0<p<1$ ) the following disjunctive formulation of $(2.3)$ :

$$
\begin{aligned}
\min & c^{T} x \\
\text { subject to } & T x \in \bigcup_{j \in J} K_{j}, \\
& x \in \mathcal{D} .
\end{aligned}
$$

Its main advantage is an insight into the nature of the non-convexity of the feasible set. In particular, we can formulate the following necessary and sufficient condition for the existence of an optimal solution of (2.4).

Assumption 2.5. The set $\Lambda:=\left\{(u, w) \in \mathbb{R}_{+}^{m+s} \mid A^{T} w+T^{T} u \leq c\right\}$ is nonempty.

Theorem 2.6. Assume that the feasible set of (2.4) is nonempty. Then (2.4) has an optimal solution if and only if Assumption 2.5 holds.

Proof. If (2.4) has an optimal solution, then for some $j \in J$ the linear program

$$
\begin{aligned}
\min & c^{T} x \\
\text { subject to } & T x \geq v^{j} \\
& A \geq b \\
& x \geq 0
\end{aligned}
$$

has an optimal solution. By duality in linear programming, its dual

$$
\begin{aligned}
\max & \left(v^{j}\right)^{T} u+b^{T} w \\
\text { subject to } & T^{T} u+A^{T} w \leq c \\
& u, w \geq 0
\end{aligned}
$$

has an optimal solution and the optimal values of both programs are equal. Thus, Assumption 2.5 must hold. On the other hand, if Assumption 2.5 is satisfied, all dual programs (2.6) for $j \in J$ have nonempty feasible sets, so the objective values of all primal problems (2.5) are bounded from below. Since one of them has a nonempty feasible set by assumption, an optimal solution must exist.

A straightforward way to solve (1.1) is to find all p-efficient points and to process all corresponding problems (2.5) (an example of such an approach is presented in [17]). Specialized bounding-pruning techniques can be used to avoid solving all of them. For example, any feasible solution $(\tilde{u}, \tilde{w})$ of the dual (2.6) can be used to generate a lower bound for (2.5). If it is worse than the best solution found so far, we can delete the problem (2.5); otherwise it has to be included into a list of problems to be solved exactly.

For multi-dimensional random vectors $\xi$ the number of $p$-efficient points can be very large and their straightforward enumeration - very difficult. It would be desirable, therefore, to avoid the complete enumeration and to search for promising $p$-efficient points only. We shall return to this issue in section 5 .

In the case of independent subvectors, $p$-efficient points have a specific structure. 
Lemma 2.7. Let $\xi$ be an s-dimensional integer random vector and let $p \in(0,1)$. Assume that $\xi=\left(\xi^{1}, \ldots, \xi^{L}\right)$, where the $s_{l^{-}}$dimensional subvectors $\xi^{l}, l=1, \ldots, L$, are independent $\left(\sum_{l=1}^{L} s_{l}=s\right)$. A vector $v=\left(v^{1}, \ldots, v^{L}\right)$, where $v^{l} \in \mathbb{Z}^{s_{l}}, l=1, \ldots, L$, is a p-efficient point of the distribution function $F$ of $\xi$ if and only if there exist $p_{l} \in(0,1]$ with $\prod_{l=1}^{L} p_{l}=p$ such that each $v^{l}$ is $p_{l^{-}}$efficient for the corresponding marginal distribution function $F_{l}\left(z^{l}\right)=\mathbb{P}\left\{\xi^{l} \leq z^{l}\right\}, l=1, \ldots, L$.

Proof. Let $v$ be $p$-efficient. Define $p_{l}^{\max }=\mathbb{P}\left\{\xi^{l} \leq v^{l}\right\}$. By independence, $\mathbb{P}\{\xi \leq v\}=$ $\Pi_{l=1}^{L} p_{l}^{\max } \geq p$. Each $v^{l}$ is $p_{l}^{\max }$-efficient for $F_{l}$, since otherwise $v$ would not be $p$-efficient. If $\Pi_{l=1}^{L} p_{l}^{\max }=p$, we are done. Otherwise, let $p_{l}^{\min }=\max \left\{F_{l}\left(z^{l}\right): z^{l} \in \mathbb{Z}^{s_{l}}, z^{l} \leq v^{l}, z^{l} \neq v^{l}\right\}$. Since $v$ is $p$-efficient, $\Pi_{l=1}^{L} p_{l}^{\min }<p$. Consequently, we can choose $p_{l} \in\left(p_{l}^{\min }, p_{l}^{\max }\right]$ such that $\prod_{l=1}^{L} p_{l}=p$ and each $v^{l}$ is still $p_{l}$-efficient.

The opposite implication is obvious.

\section{$3 \quad r$-Concave Discrete Distribution Functions}

Since the set $\mathcal{Z}_{p}$ need not be convex, it is essential to analyse its properties and to find equivalent formulations with more convenient structures. To this end we shall recall and adapt the notion of $r$-concavity of a distribution function. It uses the generalized mean function $m_{r}: \mathbb{R}_{+} \times \mathbb{R}_{+} \times[0,1] \rightarrow \mathbb{R}$ defined as follows:

$$
m_{r}(a, b, \lambda)=0 \quad \text { for } \quad a b=0,
$$

and if $a>0, b>0,0 \leq \lambda \leq 1$, then

$$
m_{r}(a, b, \lambda)=\left\{\begin{array}{cl}
a^{\lambda} b^{1-\lambda} & \text { if } r=0, \\
\max \{a, b\} & \text { if } r=\infty, \\
\min \{a, b\} & \text { if } r=-\infty \\
\left(\lambda a^{r}+(1-\lambda) b^{r}\right)^{1 / r} & \text { otherwise. }
\end{array}\right.
$$

Definition 3.1. A distribution function $F: \mathbb{R}^{s} \rightarrow[0,1]$ is called $r$-concave, where $r \in$ $[-\infty, \infty]$, if

$$
F(\lambda x+(1-\lambda) y) \geq m_{r}(F(x), F(y), \lambda)
$$

for all $x, y \in \mathbb{R}^{s}$ and all $\lambda \in[0,1]$,

If $r=-\infty$ we call $F$ quasi-concave, for $r=0$ it is known as log-concave, and for $r=1$ the function $F$ is concave in the usual sense.

The concept of a log-concave probability measure (the case $r=0$ ) was introduced and studied in $[22,23]$. The notion of $r$-concavity and corresponding results were given in $[4,5]$. For detailed description and proofs, see [26].

By monotonicity, $r$-concavity of a distribution function is equivalent to the inequality

$$
F(z) \geq m_{r}(F(x), F(y), \lambda)
$$

for all $z \geq \lambda x+(1-\lambda) y$. 
Clearly, distribution functions of integer random variables are not continuous, and cannot be $r$-concave in the sense of the above definition. Therefore, we relax Definition 3.1 in the following way.

Definition 3.2. A distribution function $F$ is called $r$-concave on the set $\mathcal{A} \subset \mathbb{R}^{s}$ with $r \in[-\infty, \infty]$, if

$$
F(z) \geq m_{r}(F(x), F(y), \lambda)
$$

for all $z, x, y \in \mathcal{A}$ and $\lambda \in(0,1)$ such that $z \geq \lambda x+(1-\lambda) y$.

To illustrate the relation between the two definitions let us consider the case of integer random vectors which are roundups of continuously distributed random vectors.

Remark 3.3. If the distribution function of a random vector $\eta$ is $r$-concave on $\mathbb{R}^{s}$ then the distribution function of $\xi=\lceil\eta\rceil$ is $r$-concave on $\mathbb{Z}^{s}$.

The last property follows from the observation that at integer points both distribution functions coincide. For the relations between the $r$-concavity of the distribution function of $\eta$ and the $r$-concavity of its density the Reader is referred to [4, 5, 28].

The concept of $r$-concavity on a set can be used to find an equivalent representation of the set $\mathcal{Z}_{p}$ given by (2.2).

Theorem 3.4. Let $\mathcal{Z}$ be the set of all possible values of an integer random vector $\xi$. If the distribution function $F$ of $\xi$ is $r$-concave on $\mathcal{Z}+\mathbb{Z}_{+}^{s}$, for some $r \in[-\infty, \infty]$, then for every $p \in(0,1)$ one has

$$
\mathcal{Z}_{p}=\left\{y \in \mathbb{R}^{s}: y \geq z \geq \sum_{j \in J} \lambda_{j} v^{j}, \sum_{j \in J} \lambda_{j}=1, \lambda_{j} \geq 0, z \in \mathbb{Z}^{s}\right\}
$$

where $v^{j}, j \in J$, are the $p$-efficient points of $F$.

Proof. By the monotonicity of $F$ we have $F(y) \geq F(z)$ if $y \geq z$. It is, therefore, sufficient to show that $\mathbb{P}(\xi \leq z) \geq p$ for all $z \in \mathbb{Z}^{s}$ such that $z \geq \sum_{j \in J} \lambda_{j} v^{j}$ with $\lambda_{j} \geq 0$, $\sum_{j \in J} \lambda_{j}=1$. We consider five cases with respect to $r$.

Case 1: $r=\infty$. It follows from the definition of $r$-concavity that $F(z) \geq \max \left\{F\left(v^{j}\right), j \in\right.$ $\left.J: \lambda_{j} \neq 0\right\} \geq p$.

Case 2: $r=-\infty$. Since $F\left(v^{j}\right) \geq p$ for each index $j \in J$ such that $\lambda_{j} \neq 0$, the assertion follows as in Case 1.

Case 3: $r=0$. By the definition of $r$-concavity,

$$
F(z) \geq \prod_{j \in J}\left[F\left(v^{j}\right)\right]^{\lambda_{j}} \geq \prod_{j \in J} p^{\lambda_{j}}=p
$$


Case 4: $r \in(-\infty, 0)$. By the definition of $r$-concavity,

$$
[F(z)]^{r} \leq \sum_{j \in J} \lambda_{j}\left[F\left(v^{j}\right)\right]^{r} \leq \sum_{j \in J} \lambda_{j} p^{r}=p^{r}
$$

Since $r<0$, we obtain $F(z) \geq p$.

Case 5: $r \in(0, \infty)$. By the definition of $r$-concavity,

$$
[F(z)]^{r} \geq \sum_{j \in J} \lambda_{j}\left[F\left(v^{j}\right)\right]^{r} \geq \sum_{j \in J} \lambda_{j} p^{r}=p^{r}
$$

For example, the set $\mathcal{Z}_{p}$ illustrated in Figure 2.1 cannot correspond to any $r$-concave distribution function, because its convex hull contains integer points which do not belong to $\mathcal{Z}_{p}$, namely, the points $(3,6),(4,5)$ and $(6,2)$.

Under the conditions of Theorem 3.4, problem (2.4) can be formulated in the following equivalent way:

$$
\begin{array}{ll}
\min & c^{T} x \\
\text { subject to } & x \in \mathcal{D} \\
& T x \geq z, \\
& z \in \mathbb{Z}^{s}, \\
& z \geq \sum_{j \in J} \lambda_{j} v^{j} \\
& \sum_{j \in J} \lambda_{j}=1 \\
& \lambda_{j} \geq 0, j \in J .
\end{array}
$$

So, the probabilistic constraint has been replaced by linear equations and inequalites, together with the integrality requirement (3.4). This condition cannot be dropped, in general. However, if other conditions of the problem imply that $T x$ is integer (for example, we have an additional constraint in the definition of $\mathcal{D}$ that $x \in \mathbb{Z}^{n}$, and $T$ has integer entries), we may dispose of $z$ totally, and replace constraints (3.3)-(3.5) with

$$
T x \geq \sum_{j \in J} \lambda_{j} v^{j}
$$

If $\xi$ takes values on a non-uniform grid, condition (3.4) should be replaced by the requirement that $z$ is a grid point.

The difficulty comes from the implicitly given $p$-efficient points $v_{j}, j \in J$. Our objective will be to avoid their enumeration and to develop an approach that generates them only when needed.

An obvious question arises: which distributions are $r$-concave in our sense? We devote the remaining part of this section to some useful observations on this topic.

Directly from the definition and Hölder's inequality we obtain the following property. 
Remark 3.5. If a distribution function $F$ is $r$-concave on the set $\mathcal{A} \subset \mathbb{R}^{s}$ with some $r \in[-\infty, \infty]$, then it is $\rho$-concave on $\mathcal{A}$ for all $\rho \in[-\infty, r]$.

For binary random vectors we have the strongest possible property.

Proposition 3.6. Every distribution function of an s-dimensional binary random vector is $r$-concave on $\mathbb{Z}_{+}^{s}$ for all $r \in[-\infty, \infty]$.

Proof. Let $x, y \in \mathbb{Z}_{+}^{s}, \lambda \in(0,1)$ and let $z \geq \lambda x+(1-\lambda) y$. By projecting $x$ and $y$ on $\{0,1\}^{s}$ we get some $x^{\prime}$ and $y^{\prime}$ such that $F\left(x^{\prime}\right)=F(x), F\left(y^{\prime}\right)=F(y)$ and $z \geq$ $\lambda x^{\prime}+(1-\lambda) y^{\prime}$. Since $z$ is integer and $x^{\prime}$ and $y^{\prime}$ binary, then $z \geq x^{\prime}$ and $z \geq y^{\prime}$. Thus $F(z) \geq \max \left(F\left(x^{\prime}\right), F\left(y^{\prime}\right)\right)=\max (F(x), F(y))$. Consequently, $F$ is $\infty$-concave and the result follows from Remark 3.5.

For scalar integer random variables our definition of $r$-concavity is related to log-concavity of sequences. A sequence $p_{k}, k=\ldots,-1,0,1, \ldots$, is called log-concave, if $p_{k}^{2} \geq p_{k-1} p_{k+1}$ for all $k$. By [10] (see also [26, Thm. 4.7.2]) and Remark 3.5, we have the following property.

Proposition 3.7. Suppose that for a scalar integer random variable $\xi$ the probabilities $p_{k}=\mathbb{P}\{\xi=k\}, k=\ldots,-1,0,1, \ldots$, form a log-concave sequence. Then the distribution function of $\xi$ is $r$-concave on $\mathbb{Z}$ for every $r \in[-\infty, 0]$.

Many well-known one-dimensional discrete distributions satisfy the conditions of Proposition 3.7: the Poisson distribution, the geometrical distribution, the binomial distribution [26, p. 109].

We end this section with sufficient conditions for the $r$-concavity of the joint distribution function in the case of integer-valued independent subvectors. Our assertion, presented in the next proposition is the discrete version of an observation from [19]. The same proof, using Hölder's inequality, works in our case as well.

Proposition 3.8. Assume that $\xi=\left(\xi^{1}, \ldots, \xi^{L}\right)$, where the $s_{l}$-dimensional subvectors $\xi_{l}$, $i=l, \ldots, L$, are independent $\left(\sum_{l=1}^{L} s_{l}=s\right)$. Furthermore, let the marginal distribution functions $F_{l}: \mathbb{R}^{s_{l}} \rightarrow[0,1]$ be $r_{l}$-concave on sets $\mathcal{A}_{l} \subset \mathbb{Z}^{s_{l}}$.

(i) If $r_{l}>0, l=1, \ldots, L$, then $F$ is $r$-concave on $\mathcal{A}=\mathcal{A}_{1} \times \cdots \times \mathcal{A}_{L}$ with

$$
r=\left(\sum_{l=1}^{L} r_{l}^{-1}\right)^{-1}
$$

(ii) If $r_{l}=0, l=1, \ldots, L$, then $F$ is log-concave on $\mathcal{A}=\mathcal{A}_{1} \times \cdots \times \mathcal{A}_{L}$.

\section{Lagrangian Relaxation}

Let us split variables in problem (2.3):

$$
\begin{aligned}
\min & c^{T} x \\
& T x=z, \\
& x \in \mathcal{D}, \\
& z \in \mathcal{Z}_{p} .
\end{aligned}
$$


Associating Lagrange multipliers $u \in \mathbb{R}^{s}$ with constraints (4.1) we obtain the Lagrangian function:

$$
L(x, z, u)=c^{T} x+u^{T}(z-T x) .
$$

Owing to the structure of $\mathcal{Z}_{p}$ (Lemma 2.2), we could have replaced equality $T x=z$ in (4.1) by an inequality $T x \geq z$, and use $u \geq 0$ in the Lagrangian. However, formal splitting (4.1) leads to the same conclusion. The dual functional has the form

$$
\Psi(u)=\inf _{(x, z) \in \mathcal{D} \times \mathcal{Z}_{p}} L(x, z, u)=h(u)+d(u)
$$

where

$$
\begin{aligned}
& h(u)=\inf \left\{\left(c-T^{T} u\right)^{T} x \mid x \in \mathcal{D}\right\}, \\
& d(u)=\inf \left\{u^{T} z \mid z \in \mathcal{Z}_{p}\right\} .
\end{aligned}
$$

Lemma 4.1. $\operatorname{dom} \Psi=\left\{u \in \mathbb{R}_{+}^{s}\right.$ : there exists $w \in \mathbb{R}_{+}^{m}$ such that $\left.A^{T} w+T^{T} u \leq c\right\}$.

Proof. Clearly, $\operatorname{dom} \Psi=\operatorname{dom} h \cap \operatorname{dom} d$. Let us calculate dom $h$. The recession cone of $\mathcal{D}$,

$$
C=\left\{y \in \mathbb{R}^{n}: A y \geq 0, y \geq 0\right\}
$$

has the dual cone

$$
C^{*}=\left\{v \in \mathbb{R}^{m}: v^{T} y \geq 0 \text { for all } y \in C\right\}=\left\{v \in \mathbb{R}^{m}: v \geq A^{T} w, w \geq 0\right\},
$$

as follows from Farkas' lemma. Thus

$$
\operatorname{dom} h=\left\{u \in \mathbb{R}^{s}: c-T^{T} u \in C^{*}\right\}=\left\{u \in \mathbb{R}^{s}: T^{T} u+A^{T} w \leq c, w \geq 0\right\} .
$$

On the other hand, by Remark 2.4 , dom $d=\mathbb{R}_{+}^{s}$, and the result follows.

The lemma implies that Assumption 2.5, which is necessary and sufficient for the existence of solutions, is also necessary and sufficient for the nonemptiness of the domain of the dual functional.

For any $u \in \mathbb{R}_{+}^{s}$ the value of $\Psi(u)$ is a lower bound on the optimal value $F^{*}$ of the original problem. This is true for all problems of form (1.1), irrespective whether the distribution function of $\xi$ is or is not $r$-concave.

The best Lagrangian lower bound will be given by

$$
D^{*}=\sup \Psi(u) \text {. }
$$

If an optimal solution of (2.3) exists, then Assumption 2.5 holds, so, by Lemma 4.1,

$$
-\infty<D^{*} \leq F^{*}
$$


We shall show that the supremum $D^{*}$ is attained. Indeed, $h(u)=-\delta_{\mathcal{D}}^{*}\left(-c+T^{T} u\right)$, where $\delta_{\mathcal{D}}^{*}(\cdot)$ is the support function of $\mathcal{D}$. Thus $h(\cdot)$ is concave and polyhedral (see [29], Corollary 19.2.1). By Remark 2.4, for $u \geq 0$ the minimization in (4.3) may be restricted to finitely many $p$-efficient points $v^{j}, j \in J$. For $u \nsupseteq 0$ one has $d(u)=-\infty$. Therefore, $d(\cdot)$ is concave and polyhedral as well. Consequently, $\Psi(\cdot)$ is concave and polyhedral. Since it is bounded from above by $F^{*}$, it must attain its maximum.

Another lower bound may be obtained from the convexification of problem (2.3)

$$
F_{\text {co }}^{*}=\min \left\{c^{T} x \mid T x=z, x \in \mathcal{D}, z \in \operatorname{co} \mathcal{Z}_{p}\right\} .
$$

It is known (see [18]) that

$$
F_{\text {co }}^{*}=D^{*} \leq F^{*}
$$

We now analyse in more detail the structure of the dual functional $\Psi$. Let us start from $h(\cdot)$. If Assumption 2.5 is satisfied, then for each $u \in \mathbb{R}^{s}$

$$
h(u)=\sup \left\{b^{T} w \mid T^{T} u+A^{T} w \leq c, w \geq 0\right\},
$$

according to the duality theory in linear programming. This allows us to reformulate the dual problem (4.4) in a more explicit way:

$$
\begin{array}{ll}
\max & d(u)+b^{T} w \\
& T^{T} u+A^{T} w \leq c, \\
& u \geq 0, w \geq 0 .
\end{array}
$$

Let us observe that we may write 'max' instead of 'sup' because we already know that the supremum is attained. We may also add the constraint ' $u \geq 0$ ' explicitly, since it defines the domain of $d$.

Properties of $d(\cdot)$ can also be analysed in a more explicit way.

Lemma 4.2. For every $u \geq 0$ the solution set of the subproblem

$$
\min _{z \in \mathcal{Z}_{p}} u^{T} z
$$

is nonempty and has the following form:

$$
\hat{Z}(u)=\bigcup_{j \in \hat{J}(u)}\left\{v^{j}\right\}+C(u),
$$

where $\hat{J}(u)$ is the set of $p$-efficient solutions of (4.9), and

$$
C(u)=\left\{d \in \mathbb{R}_{+}^{s}: d_{i}=0 \text { if } u_{i}>0, i=1, \ldots, s\right\} .
$$

Proof. The result follows from Remark 2.4. Let us at first consider the case $u>0$. Suppose that a solution $z$ to (4.9) is not a $p$-efficient point. Then there is a $p$-efficient $v \in \mathcal{Z}_{p}$ such that $v \leq z$, so $u^{T} v<u^{T} z$, a contradiction. Thus, for all $u \geq 0$ all solutions to (4.9) are $p$-efficient. In the general case $u \geq 0$, if a solution $z$ is not $p$-efficient, we must have $u^{T} v=u^{T} z$ for all $p$-efficient $v \leq z$. This is equivalent to $z \in\{v\}+C(u)$, as required. 
The last result allows us to calculate the subdifferential of $d$ in a closed form.

Lemma 4.3. For every $u \geq 0$ one has $\partial d(u)=\operatorname{co}\left\{v^{j}, j \in \hat{J}(u)\right\}+C(u)$.

Proof. From (4.3) it follows that

$$
d(u)=-\delta_{\mathcal{Z}_{p}}^{*}(-u),
$$

where $\delta_{\mathcal{Z}_{p}}^{*}(\cdot)$ is the support function of $\mathcal{Z}_{p}$ and, consequently, of co $\mathcal{Z}_{p}$. This fact follows from the structure of $\mathcal{Z}_{p}$ (Remark 2.4) by virtue of Corolarry 16.5.1 in [29]. By [29, Thm 23.5], $g \in \partial \delta_{\mathcal{Z}_{p}}^{*}(-u)$ if and only if $\delta_{\mathcal{Z}_{p}}^{*}(-u)+\delta_{\text {co }} \mathcal{Z}_{p}(g)=-g^{T} u$, where $\delta_{\text {co }} \mathcal{Z}_{p}(\cdot)$ is the indicator function of co $\mathcal{Z}_{p}$. It follows that $g \in \operatorname{co} \mathcal{Z}_{p}$ and $\delta_{\mathcal{Z}_{p}}^{*}(-u)=-g^{T} u$. Thus, $g$ is a convex combination of solutions to (4.9) and the result follows from Lemma 4.2.

Therefore the following necessary and sufficient optimality conditions for problem (4.6)-(4.8) can be formulated.

Theorem 4.4. A pair $(u, w) \in \Lambda$ is an optimal solution of (4.6)-(4.8) if and only if there exists a point $x \in \mathbb{R}_{+}^{n}$ such that:

$$
A x \geq b, \quad w^{T}(A x-b)=0,
$$

and

$$
T x \in \operatorname{co}\left\{v^{j}: j \in \hat{J}(u)\right\}+C(u),
$$

where $\hat{J}(u)$ is the set of p-efficient solutions of (4.9), and $C(u)$ is given by (4.10).

Proof. The vector $x$ plays the role of the Lagrange multiplier associated with the constraint (4.7). The necessary condition of optimality for (4.6)-(4.8) (Kuhn-Tucker condition) has the form

$$
\partial\left(b^{T} w+d(u)+x^{T}\left(c-T^{T} u-A^{T} w\right)\right) \cap K(u, w) \neq \emptyset,
$$

where $K(u, w)$ is the normal cone to $\mathbb{R}_{+}^{m+s}$ at $(u, w)$. Using the closed-form expression for the subdifferential of $d$ from Lemma 4.3, we obtain:

$$
\partial\left(b^{T} w+d(u)+x^{T}\left(c-T^{T} u-A^{T} w\right)\right)=\left(\begin{array}{l}
\operatorname{co}\left\{v^{j}: j \in \hat{J}(u)\right\}+C(u)-T x \\
b-A x
\end{array}\right) .
$$

On the other hand:

$$
K(u, w)=\left\{\left(u^{*}, w^{*}\right): u^{*} \leq 0, w^{*} \leq 0,\left\langle u^{*}, u\right\rangle=0,\left\langle w^{*}, w\right\rangle=0\right\}=\left(\begin{array}{l}
-C(u) \\
-C(w)
\end{array}\right) .
$$

Consequently, the condition co $\left\{v^{j}: j \in \hat{J}(u)\right\}+C(u)-T x \cap-C(u) \neq \emptyset$ implies the existence of elements $v \in \operatorname{co}\left\{v^{j}: j \in \hat{J}(u)\right\}$ and $c_{1}, c_{2} \in C(u)$ such that: $v+c_{1}-T x=-c_{2}$, which is equivalent to the condition (4.12). Furthermore, we obtain that $b-A x \cap-C(w) \neq$ $\emptyset$. The definition of $C(w)$ implies condition (4.11). 
It follows that the optimal Lagrangian bound is associated with a certain primal solution $x$ which is feasible with respect to the deterministic constraints and such that $T x \in \operatorname{co} \mathcal{Z}_{p}$. Moreover, since $(u, w) \in \Lambda$, the point $x$ is optimal for the convex hull problem:

$$
\begin{array}{ll}
\min & c^{T} x \\
& A x \geq b, \\
& T x \geq \sum_{j \in J} \lambda_{j} v^{j}, \\
& \sum_{j \in J} \lambda_{j}=1, \\
& x \geq 0, \quad \lambda \geq 0 .
\end{array}
$$

Indeed, associating with (4.14) multipliers $w$, with (4.15) multipliers $u$, and with (4.16) a multiplier $\mu=d(u)$, we can show that $(x, \bar{\lambda})$ is optimal for $(4.13)-(4.17)$ provided that $\bar{\lambda}_{j}$ are the coefficients at $v^{j}$ in the convex combination in (4.12).

Since the set of $p$-efficient points is not known, we need a numerical method for solving (4.6)-(4.8) or its dual (4.13)-(4.17).

Let us stress that all considerations of this section apply to non-uniform grids $\mathcal{Z}$. The same is true for the method to be presented in the next section.

\section{The cone generation method}

The idea of a numerical method for calculating Lagrangian bounds is embedded in the convex hull formulation (4.13)-(4.17). We shall develop for it a new specialized method, which separates the generation of $p$-efficient points and the solution of the approximation of the original problem using these points. It is related to column generation methods, which have been known since the classical work [11] as extremely useful tools of large scale linear and integer programming $[2,8]$.

\section{The Method}

Step 0: Select a $p$-efficient point $v^{0}$. Set $J_{0}=\{0\}, k=0$.

Step 1: Solve the master problem

$$
\begin{array}{ll}
\min & c^{T} x \\
& A x \geq b, \\
& T x \geq \sum_{j \in J_{k}} \lambda_{j} v^{j}, \\
& \sum_{j \in J_{k}} \lambda_{j}=1, \\
& x \geq 0, \quad \lambda \geq 0 .
\end{array}
$$

Let $u^{k}$ be the vector of simplex multipliers associated with the constraint (5.3). 
Step 2: Calculate an upper bound for the dual functional:

$$
\bar{d}\left(u^{k}\right)=\min _{j \in J_{k}}\left(u^{k}\right)^{T} v^{j} .
$$

Step 3: Find a $p$-efficient solution $v^{k+1}$ of the subproblem:

$$
\min _{z \in \mathcal{Z}_{p}}\left(u^{k}\right)^{T} z
$$

and calculate

$$
d\left(u^{k}\right)=\left(v^{k+1}\right)^{T} u^{k}
$$

Step 4: If $d\left(u^{k}\right)=\bar{d}\left(u^{k}\right)$ then stop; otherwise set $J_{k+1}=J_{k} \cup\{k+1\}$, increase $k$ by one and go to Step 1.

A few comments are in order. The first $p$-efficient point $v^{0}$ can be found by solving (4.9) for an arbitrary $u \geq 0$. All master problems will be solvable, if the first one is solvable, i.e., if the set $\left\{x \in \mathbb{R}_{+}^{n}: A x \geq b, T x \geq v^{0}\right\}$ is nonempty. If not, adding a penalty term $M \mathbb{1}^{T} t$ to the objective, and replacing (5.3) by

$$
T x+t \geq \sum_{j \in J_{k}} \lambda_{j} v^{j},
$$

with $t \geq 0$ and a very large $M$, is the usual remedy $\left(\mathbb{1}^{T}=\left[\begin{array}{llll}1 & 1 & \ldots & 1\end{array}\right]\right)$. The calculation of the upper bound at Step 2 is easy, because one can simply select $j_{k} \in J_{k}$ with $\lambda_{j_{k}}>0$ and set $\bar{d}\left(u^{k}\right)=\left(u^{k}\right)^{T} v^{j_{k}}$. At Step 3 one may search for $p$-efficient solutions only, due to Lemma 4.2 .

The algorithm is finite. Indeed, the set $J_{k}$ cannot grow indefinitely, because there are finitely many $p$-efficient points (Theorem 2.3). If the stopping test of Step 4 is satisfied, optimality conditions for (4.13)-(4.17) are satisfied. Moreover $\hat{J}_{k}=\left\{j \in J_{k}:\left\langle v^{j}, u^{k}\right\rangle=\right.$ $\left.d\left(u^{k}\right)\right\} \subseteq \hat{J}(u)$

When the dimension of $x$ is large and the number of rows of $T$ small, an attractive alternative to the cone generation method is provided by bundle methods applied directly to the dual problem

$$
\max _{u \geq 0}[h(u)+d(u)]
$$

because at any $u \geq 0$ subgradients of $h$ and $d$ are readily available. For a comprehensive description of bundle methods the reader is refereed to $[13,14]$. It may be interesting to note that in our case they correspond to a version of the augmented Lagrangian method (see $[30,31])$.

Let us now focus our attention on solving the auxiliary problem (4.9), which is explicitly written as:

$$
\min \left\{u^{T} z \mid F(z) \geq p\right\}
$$


where $F(\cdot)$ denotes the distribution function of $\xi$.

Assume that the components $\xi_{i}, i=1, \ldots, s$, are independent. Then we can write the probabilistic constraint in the following form:

$$
\ln (F(z))=\sum_{i=1}^{s} \ln \left(F_{i}\left(z_{i}\right)\right) \geq \ln p .
$$

Since we know that at least one of the solutions is a $p$-efficient point, with no loss of generality we may restrict the search to grid vectors $z$. Furthermore, by Lemma 2.2, we have $z_{i} \geq l_{i}$, where $l_{i}$ are $p$-efficient points of $\xi_{i}$. For integer grids we obtain a nonlinear knapsack problem:

$$
\begin{aligned}
\min & \sum_{i=1}^{s} u_{i} z_{i} \\
& \sum_{i=1}^{s} \ln \left(F_{i}\left(z_{i}\right)\right) \geq \ln p, \\
& z_{i} \geq l_{i}, \quad z_{i} \in \mathbb{Z}, \quad i=1, \ldots, s .
\end{aligned}
$$

If $b_{i}$ is a known upper bound on $z_{i}, i=1, \ldots, s$, we can transform the above problem to a $0-1$ linear programming problem:

$$
\begin{aligned}
\min & \sum_{i=1}^{s} \sum_{j=l_{i}}^{b_{i}} j u_{i} y_{i j} \\
& \sum_{\substack{i=1 \\
b_{i}}}^{b_{i}} \sum_{j=l_{i}}^{b_{i}} \ln \left(F_{i}(j)\right) y_{i j} \geq \ln p \\
& \sum_{j=l_{i}} y_{i j}=1, \quad i=1, \ldots, s, \\
& y_{i j} \in\{0,1\}, \quad i=1, \ldots, s, \quad j=l_{i}, \ldots, u_{i} .
\end{aligned}
$$

In this formulation, $z_{i}=\sum_{j=l_{i}}^{b_{i}} j y_{i j}$.

For log-concave marginals $F_{i}(\cdot)$ the following compact formulation is possible. Setting $z_{i}=l_{i}+\sum_{j=l_{i}+1}^{b_{i}} \delta_{i j}$ with binary $\delta_{i j}$, we can reformulate the problem as a $0-1$ knapsack problem:

$$
\begin{aligned}
\min & \sum_{i=1}^{s} \sum_{j=l_{i}+1}^{b_{i}} u_{i} \delta_{i j} \\
& \sum_{i=1}^{s} \sum_{j=l_{i}+1}^{b_{i}} a_{i j} \delta_{i j} \geq r, \\
& \delta_{i j} \in\{0,1\}, \quad i=1, \ldots, s, \quad, j=l_{i}+1, \ldots b_{i},
\end{aligned}
$$

where $a_{i j}=\ln F_{i}(j)-\ln F_{i}(j-1)$ and $r=\ln p-\ln F(l)$. Indeed, by the log-concavity, we have $a_{i, j+1} \leq a_{i j}$, so there is always a solution with nonincreasing $\delta_{i j}, j=l_{i}+1, \ldots, b_{i}$. Very efficient solution methods exist for such knapsack problems [18]. 
If the grid $\mathcal{Z}$ is not integer we can map it to integers by numbering the posssible realizations of each $\xi_{i}$ in an icreasing order.

If the components $\xi_{i}$ of $\xi$ are dependent, new specialized algorithms are needed for solving the subproblem (5.6). The advantage of the cone generation method is that we can separate the search for new $p$-efficient points (via (5.6)) and the solution of the "easy" part of the problem: the master problem (5.1)-(5.5).

\section{Primal feasible solution and upper bounds}

Let us consider the optimal solution $x^{\text {low }}$ of the convex hull problem (4.13)-(4.17) and the corresponding multipliers $\lambda_{j}$. Define $J^{\text {low }}=\left\{j \in J: \lambda_{j}>0\right\}$.

If $J^{\text {low }}$ contains only one element, the point $x^{\text {low }}$ is feasible and therefore optimal for the disjunctive formulation (2.4). If, however, there are more positive $\lambda$ 's, we need to generate a feasible point. A natural possibility is to consider the restricted disjunctive formulation:

$$
\begin{aligned}
\min & c^{T} x \\
\text { subject to } & T x \in \bigcup_{j \in J^{\text {low }}} K_{j}, \\
& x \in \mathcal{D} .
\end{aligned}
$$

It can be solved by simple enumeration of all cases for $j \in J^{\text {low }}$ :

$$
\begin{aligned}
\min & c^{T} x \\
\text { subject to } & T x \geq v^{j} \\
& x \in \mathcal{D}
\end{aligned}
$$

In general, it is not guaranteed that any of these problems has a nonempty feasible set, as the following example shows. Let $n=3, T=I$, and let there be only three $p$-efficient points: $v^{1}=(1,0,0), v^{2}=(0,1,0), v^{3}=(0,0,1)$, and two additional deterministic constraints: $x_{1} \leq 1 / 2, x_{2} \leq 1 / 2$, and $c=(0,0,1)$. The convex hull problem has $\lambda_{1}=$ $\lambda_{2}=1 / 2, \lambda_{3}=0$, but both problems (6.2) for $j=1,2$ have empty feasible sets.

To ensure that problem (6.1) has a solution, it is sufficient that the following stronger version of Assumption 2.5 holds.

Assumption 6.1. The set $\Lambda:=\left\{(u, w) \in \mathbb{R}_{+}^{m+s} \mid A^{T} w+T^{T} u \leq c\right\}$ is nonempty and bounded.

Indeed, then each of the dual problems (2.6) has an optimal solution, so by duality in linear programming each of the subproblems (6.2) has an optimal solution. We can, therefore, solve all of them and choose the best solution.

An alternative strategy would be to solve the corresponding upper bounding problem (6.2) every time a new $p$-efficient point is generated. If $U_{j}$ denotes the optimal value of (6.2), the upper bound at iteration $k$ is

$$
\bar{U}^{k}=\min _{0 \leq j \leq k} U_{j}
$$


This may be computationally efficient, especially if we solve the dual problem (2.6), in which only the objective function changes from iteration to iteration.

If the distribution function of $\xi$ is $r$-concave on the set of possible values of $\xi$, Theorem 3.4 provides an alternative formulation of the upper bound problem (6.1):

$$
\begin{array}{ll}
\min & c^{T} x \\
\text { subject to } & x \in \mathcal{D} \\
& T x \geq z, \\
& z \in \mathbb{Z}^{s}, \\
& z \geq \sum_{j \in J_{k}} \lambda_{j} v^{j}, \\
& \sum_{j \in J_{k}} \lambda_{j}=1 \\
& \lambda_{j} \geq 0, j \in J_{k} .
\end{array}
$$

Problem (6.4) is more accurate than the bound (6.3), because the set of integer $z$ dominated by convex combinations of $p$-efficient points in $J_{k}$ is not smaller than $J_{k}$. In fact, we need to solve this problem only at the end, with $J_{k}$ replaced by $J^{\text {low }}$.

\section{$7 \quad$ Numerical Illustration}

\subsection{Traffic Assignment in Telecommunication}

In Time-Division Multiple Access (TDMA) satellite communication systems the following problem arises: given a nonnegative integer $m \times m$ traffic matrix $D$ find an integer $n$, nonnegative integers $x_{1}, \ldots, x_{n}$ (time slots) and $m \times m$ permutation matrices $Q^{(1)}, \ldots, Q^{(n)}$ (switch modes) such that

$$
\sum_{i=1}^{n} Q^{(i)} x_{i} \geq D
$$

and $\sum_{i=1}^{n} x_{i}$ is minimized. Each element $d_{k l}$ of the matrix $D$ represents the demand for transmission from station $k$ to station $l$; each permutation $Q^{(i)}$ describes an assignment of senders to receivers for simultaneous transmission in a time slot $x_{i}$.

For practical reasons from among $n$ ! possible permutations some fixed subset is selected: usually $n=2 m$ and

$$
Q^{(i)}= \begin{cases}C^{i-1} I & i=1, \ldots, n, \\ C^{i-n-1} J & i=n+1, \ldots, 2 n,\end{cases}
$$

where $C^{i}$ stands for the $i$ th power of $C$ and

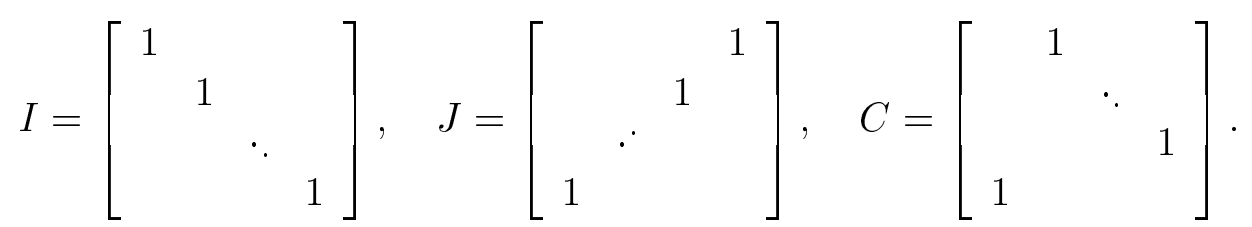


The Reader is referred to $[1,6,15]$ for the background of the TDMA problem.

If the demand $D$ is random, we obtain the probabilistically constrained problem

$$
\begin{aligned}
\min & \sum_{i=1}^{n} x_{i} \\
\text { subject to } & \mathbb{P}\left\{\sum_{i=1}^{n} Q^{(i)} x_{i} \geq D\right\} \geq p, \\
& x \geq 0, \quad x \in \mathbb{Z}^{n} .
\end{aligned}
$$

As an illustration consider the problem with $m=4$ (which makes $n=8$ ). $p=0.9$, and with independent Poisson demands having the expected values

$$
\mathbb{E}\{D\}=\left[\begin{array}{llll}
2 & 1 & 3 & 4 \\
2 & 3 & 2 & 1 \\
1 & 2 & 4 & 2 \\
3 & 2 & 1 & 3
\end{array}\right]
$$

This example problem has been solved by the cone generation method, as described in section 5. The master problem (5.1)-(5.5) (without the integrality restriction) was solved by the simplex method. The subproblem of Step 3 was formulated as a $0-1$ programming problem (5.7). This formulation turned out to be easier to solve than (5.8), which could have been used, too, due to the log-concavity of the multidimensional Poisson distribution with idependent components. The upper bounding problem (6.4) contained an additional integrality restriction on $x$.

The entire algorithm has been programmed in AMPL [12], and CPLEX was the LP/MIP solver used.

To generate the first $p$-efficient point we solved the subproblem of Step 3 with $u^{0}=$ $\left(\begin{array}{llll}1 & 1 & \ldots & 1\end{array}\right)$. This gave

$$
v^{0}=\left[\begin{array}{cccc}
5 & 4 & 7 & 9 \\
6 & 7 & 6 & 4 \\
4 & 6 & 10 & 6 \\
8 & 6 & 4 & 7
\end{array}\right]
$$

The values of the objective fuctions of the master problem, the subproblem and the upper bounding problem (6.4) at successive iterations are illustrated in Figure 7.1.

Luckily, the algorithm terminated when the roundup of the optimal value of the subproblem, $\left\lceil d\left(u^{k}\right)\right\rceil$, which is a lower bound on the optimal value of the whole problem, became equal to the optimal value of the upper bounding problem (6.4) (with integrality restrictions on $x$ ). This, of course, is not guaranteed to happen, and we might as well end at a solution with a duality gap.

By the log-concavity of the Poisson distribution (Proposition 3.7) and by Proposition 3.8 , the distribution function of $\xi$ is log-concave in the area above the expected values. Consequently, by virtue of Theorem 3.4, the optimal solution of the upper-bounding problem (6.4),

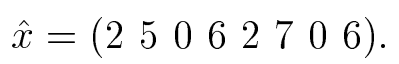




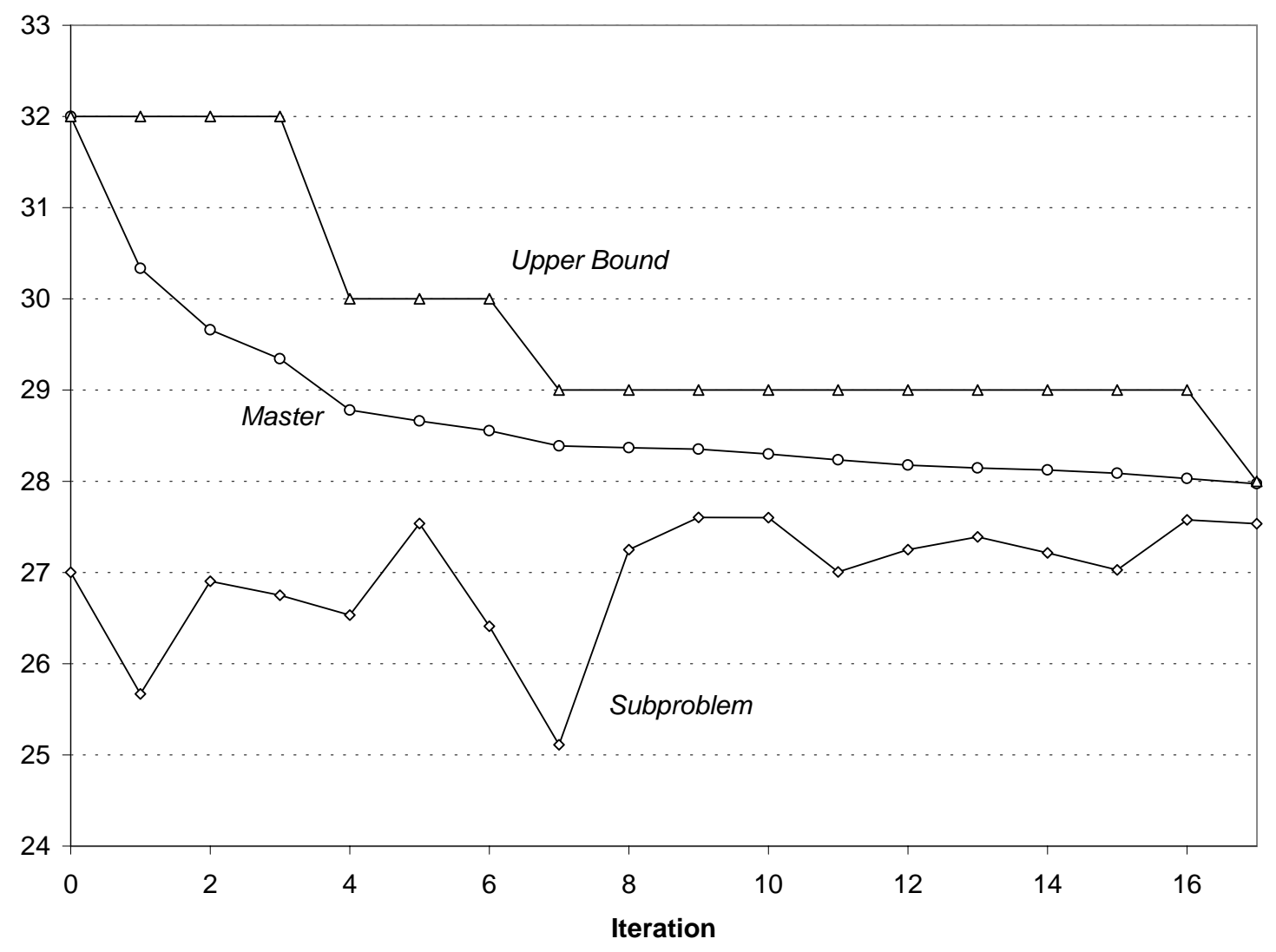

Figure 7.1: Objective values of the master problem, the subproblem and the upper bounding problem in the communication traffic assignment example.

is optimal for the original probabilistically constrained problem. The $p$-efficient point $\hat{v}$ such that $\sum_{i=1}^{n} Q^{(i)} \hat{x}_{i} \geq \hat{v}$ equals

$$
\hat{v}=\left[\begin{array}{llll}
6 & 5 & 7 & 8 \\
6 & 8 & 7 & 6 \\
7 & 7 & 8 & 5 \\
7 & 6 & 6 & 9
\end{array}\right]
$$

. It has been found on the 17 th iteration.

\subsection{Vehicle Routing}

We have a directed graph with node set $\mathcal{N}$ and arc set $\mathcal{E}$. A set of cyclic routes $\Pi$, understood as sequences of nodes connected with arcs and such that the last node of the sequence is the same as the first one, has been selected. For each $\operatorname{arc} e \in \mathcal{E}$ we denote by $\mathcal{R}(e)$ the set of routes containing $e$, and by $c(\pi)$ the unit cost on the route.

A random integer demand $\xi(e)$ is associated with each $\operatorname{arc} e \in \mathcal{E}$. Our objective is to 


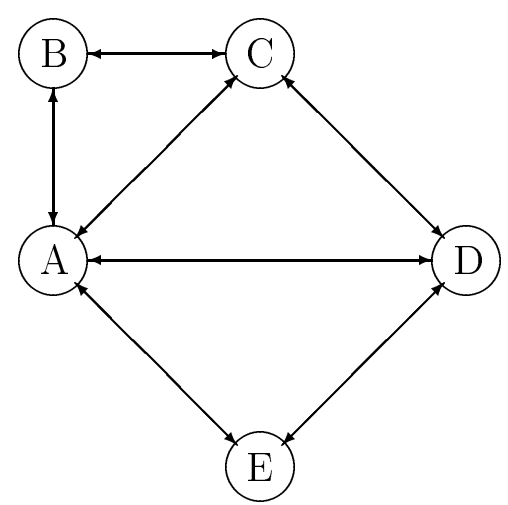

Figure 7.2: The graph of the vehicle routing problem.

find non-negative integers $x(\pi), \pi \in \Pi$, such that

$$
\mathbb{P}\left\{\sum_{\pi \in \mathcal{R}(e)} x(\pi) \geq \xi(e), e \in \mathcal{E}\right\} \geq p
$$

and the cost

$$
\sum_{p i \in \Pi} c(\pi) x(\pi)
$$

is minimized.

As an illustration, let us consider the graph shown in Figure 7.2. Each arc in this figure represents in fact two arcs in opposite directions.

We assume that demands $\xi(e)$ associated with the arcs are independent Poisson random variables with the expected values given in Table 7.1. 


\begin{tabular}{lc} 
Arc & Expected Demand \\
\hline AB & 2 \\
AC & 3 \\
AD & 2 \\
AE & 2 \\
BA & 1 \\
BC & 1 \\
CA & 2 \\
CB & 1 \\
CD & 4 \\
DA & 2 \\
DC & 4 \\
DE & 3 \\
EA & 2 \\
ED & 3
\end{tabular}

Table 7.1: Expected demands

The set of routes $\Pi$ is given by the following route-arc incidence matrix $T$ :

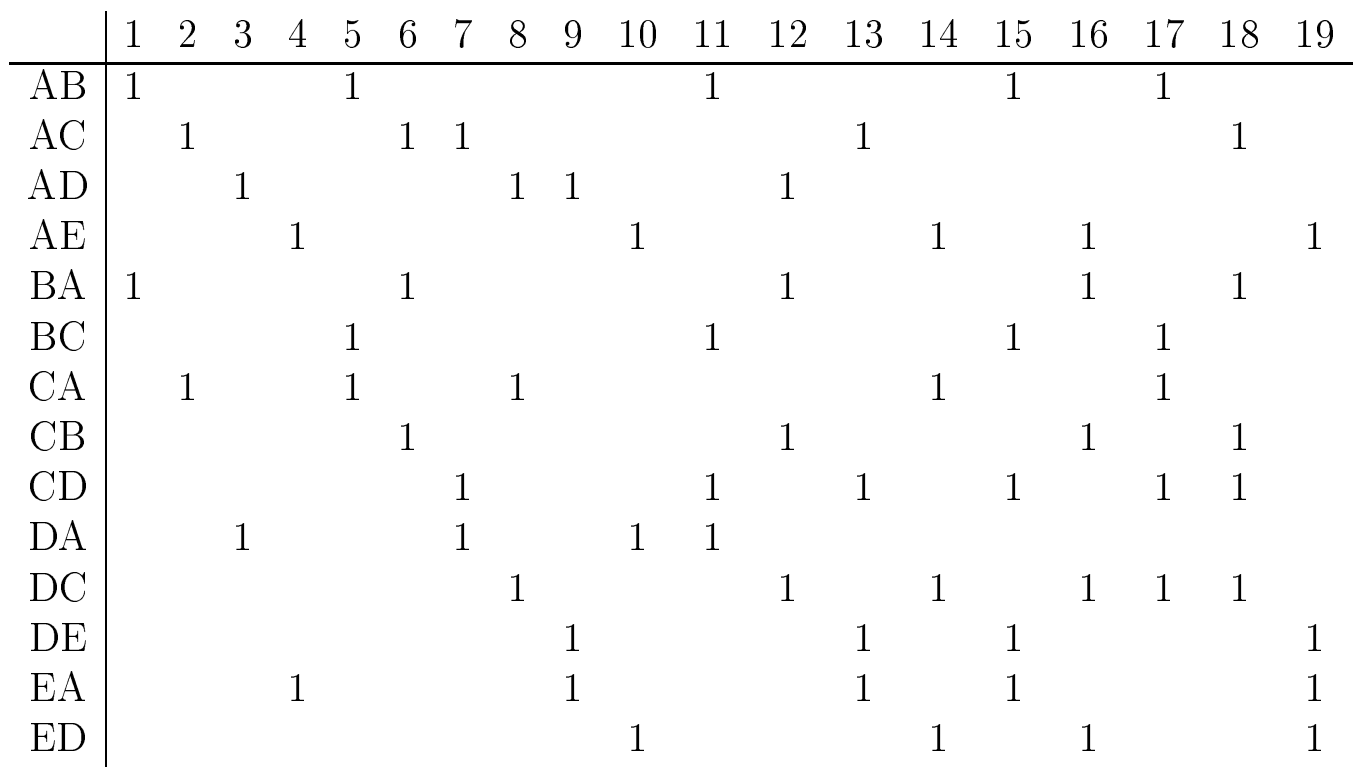

For example, route 18 has the form ACDCBA.

The cost cefficients associated with the routes are given by

$$
c=\left(\begin{array}{lll}
10151815323257576060636361617575626244
\end{array}\right) .
$$

Finally, the probability level is $p=0.9$.

Again, we used the cone generation method, implemented exactly as described in the previous example. To generate the first $p$-efficient point we solved the subproblem of 
Step 3 with $u^{0}=\left(\begin{array}{llll}1 & 1 & \ldots & 1\end{array}\right)$. This gave

$$
v^{0}=\left(\begin{array}{ll}
67664464968767)
\end{array}\right)
$$

The method terminated after 23 iterations satisfying the stopping criterion of Step 4, with the solution of the convexified problem:

$$
\hat{x}=\left(\begin{array}{llllllllllllllllll}
2 & 3 & 6 & 0 & 0 & 0 & 0 & 0 & 0 & 0 & 0 & 0 & 0 & 0 & 0 & 0 & 4 & 4
\end{array}\right),
$$

that is 2 on route ABA, 3 on ACA, 6 on ADA, 4 on ABCDCA, 4 on ACDCBA and 7 on AEDEA. The symmetry of the solution is purely accidental.

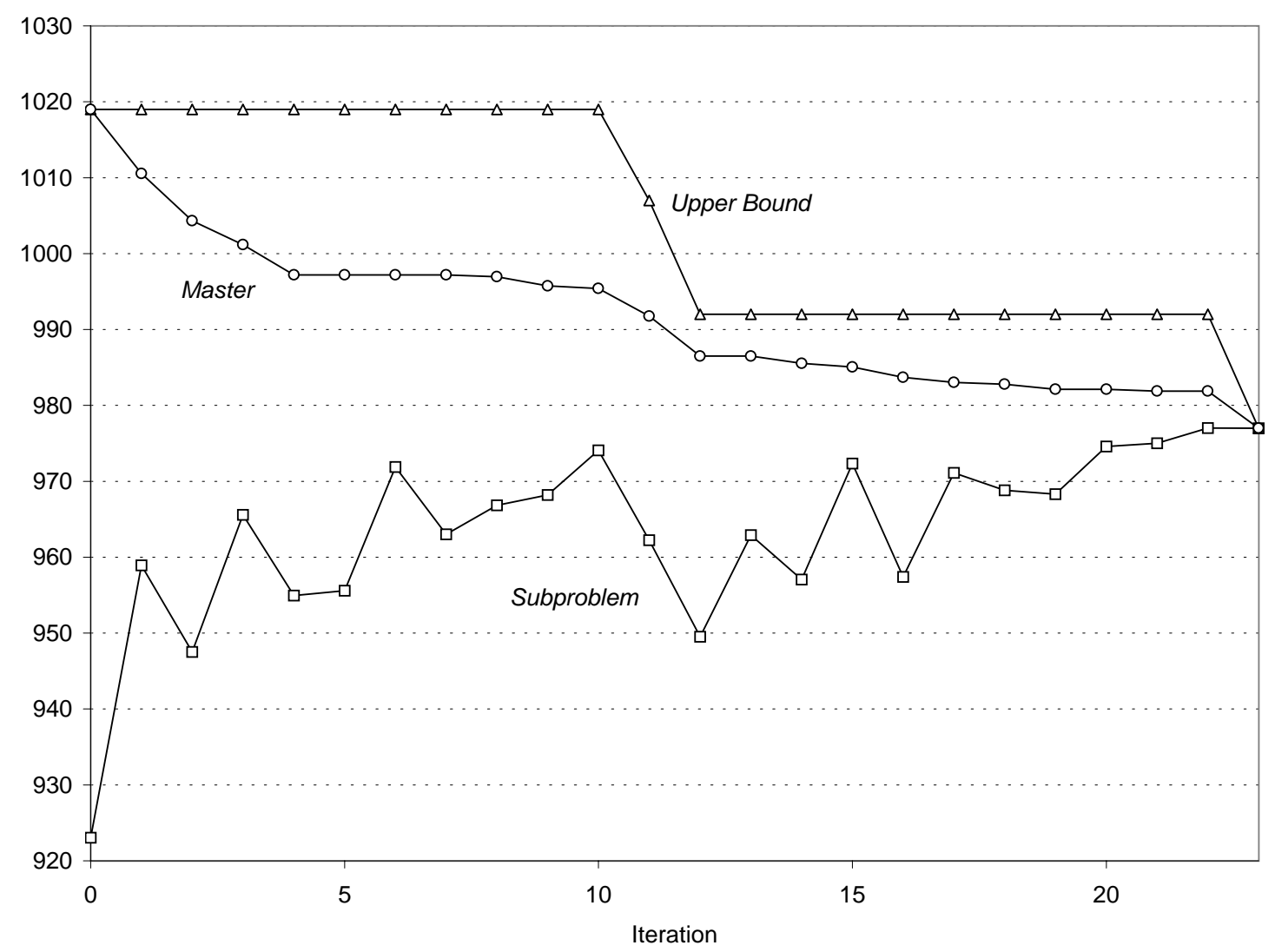

Figure 7.3: Objective values of the master problem, the subproblem and the upper bounding problem in the vehicle routing example.

The values of the objective fuctions of the master problem, the subproblem, and the upper bounding problem (6.4) in successive iterations are illustrated in Figure 7.2.

The optimal solution $\hat{x}$ of the convexified problem turned out to be integer. As in the previous example, by the log-concavity of the Poisson distribution, $\hat{x}$, as an optimal solution of the upper-bounding problem (6.4), is optimal for the original probabilistically constrained problem. In fact, we have $T \hat{x} \geq \hat{v}$, where

$$
\hat{v}=(67675474868777) \text {. }
$$


It has been found on the 22nd iteration.

\section{Acknowledgement}

The authors are grateful to two anonymous Referees for their insightful comments.

\section{References}

[1] E. Balas and P.L. Landweer, Traffic Assignment in Communication Sattelites, Operations Research Letters 2 (1983) 141-147.

[2] C. Barnhart, E.L. Johnson, G.L. Nemhauser, M.V.P. Savelsbergh and P.H. Vance, Branchand-Price: Column Generation for Solving Huge Integer Programs, Operations Research 46 (1998) 316-329.

[3] T. Becker and V. Weispfenning, Gröbner Bases, Springer-Verlag, New York, 1993.

[4] C. Borell, Convex Set Functions in d-Space, Periodica Mathematica Hungarica 6 (1975) 111-136.

[5] H.J. Brascamp and E.H. Lieb, On Extensions of the Brunn-Minkowski and PrékopaLeindler Theorems, Including Inequalities for Log-Concave Functions and with an Application to the Diffusion Equation, Journal of Functional Analysis 22 (1976) 366-389.

[6] R.E. Burkard, Time-Slot Assignment for TDMA Systems, Computing 35 (1985) 99-112. 22 (1976) 366-389.

[7] A. Charnes, W.W.Cooper and G.H. Symonds, Cost Horizons and Certainty Equivalents: An Approach to Stochastic Programming of Heating Oil, Management Science 4 (1958) 235-263.

[8] V. Chvátal, Linear Programming, W.H. Freeman, New York,, NY, 1983.

[9] C.L. Dert, Asset-Liability Management for Pension Funds: A Multistage ChanceConstrained Programming Approach, PhD Thesis, Erasmus University, Rotterdam, 1995.

[10] M. Fekete and G. Polya, Über ein Problem von Laguerre, Rediconti dei Circolo Matematico di Palermo, 23 (1912) 89-120.

[11] L.R Ford and D.R. Fulkerson, A Suggested Computation for Maximal Multicommodity Network Flows, Management Science 5 (1958) 97-101.

[12] R. Fourer, D.M. Gay and B.W. Kernighan, AMPL: A Modeling Language For Mathematical Programming, Boyd \& Fraser, Danvers, Massachusetts, 1993.

[13] J.-B. Hiriart-Urruty and C. Lemaréchal, Convex Analysis and Minimization Algorithms I and II, Springer-Verlag, Berlin 1993.

[14] K.C. Kiwiel, Methods of Descent for Nondifferentiable Optimization, Lecture Notes in Mathematics Vol. 1133, Springer-Verlag, Berlin 1985.

[15] J.L. Lewandowski, J.L. Liu and W.S. Liu, SS/TDMA Time Slot Assignment with restricted switching modes, IEEE Transactions on Communications COM-31 (1983) 149-154.

[16] L.B. Miller and H. Wagner, Chance-Constrained Programming with Joint Constraints, Operations Research 13 (1965) 930-945. 
[17] M. Murr and A. Prékopa, Solution of a Product Substitution Problem Using Stochastic Programming, RRR 32-96, RUTCOR, Rutgers Center for Operations Research, 1996.

[18] G.L. Nemhauser and L.A. Wolsey, Integer and Combinatorial Optimization, John Wiley \& Sons, New York, 1988.

[19] V.I. Norkin and N.V. Roenko, $\alpha$-Concave Functions and Measures and Their Applications, Kibernet. Sistem. Anal. 189 (1991) 77-88 (in Russian); translation in: Cybernet. Systems Anal. 27 (1991) 860-869.

[20] V.I. Norkin, Y.M. Ermoliev and A. Ruszczyński, On Optimal Allocation of Indivisibles Under Uncertainty, Operations Research 46 (1998) 381-395.

[21] A. Prékopa, On Probabilistic Constrained Programming, Proceedings of the Princeton Symposium on Mathematical Programming. Princeton University Press, 1970, pp. 113-138.

[22] A. Prékopa, Logarithmic Concave Functions with Applications to Stochastic Programming, Acta Sci. Math. (Szeged) 32 (1971) 301-316.

[23] A. Prékopa, On Logarithmic Concave Measures and Functions, Acta Sci. Math. (Szeged) 34 (1973) 339-343.

[24] A. Prékopa, Contributions to the Theory of Stochastic Programming, Mathematical Programming 4 (1973) 202-221.

[25] A. Prékopa, Dual Method for a One-Stage Stochastic Programming Problem with Random RHS Obeying a Discrete Probability Distribution, Zeitschrift für Operations Research 34 (1990) 441-461.

[26] A. Prékopa, Stochastic Programming, Kluwer, Dordrecht, Boston, 1995.

[27] A. Prékopa, B. Vizvári, and T. Badics, Programming Under Probabilistic Constraint with Discrete Random Variable, in: New Trends in Mathematical Programming, L. Grandinetti et al. (Ed.), Kluwer, Dordrecht, Boston, 1998, pp. 235-255.

[28] Y. Rinott, On Convexity of Measures, Annals of Probability 4 (1976) 1020-1026.

[29] R. T. Rockafellar, Convex Analysis, Princeton University Press, Princeton, 1970.

[30] A. Ruszczyński, A Regularized Decomposition Method for Minimizing a Sum of Polyhedral Functions, Mathematical Programming 35 (1986) 309-333.

[31] A. Ruszczyński, An Augmented Lagrangian Decomposition Method for Block Diagonal Linear Programming Problems, Operations Research Letters 8 (1989) 287-294.

[32] S. Sen, Relaxations for the Probabilistically Constrained Programs with Discrete Random Variables, Operations Research Letters 11 (1992) 81-86.

[33] M.R. Singh, C.T. Abraham and R. Akella, A Wafer Design Problem in Semiconductor Manufacturing for Reliable Customer Service, IEEE Transactions on Components, Hybrids and Manufacturing Technology 13 (1990) 103-108.

[34] S.R. Tayur, R.R. Thomas and N.R. Natraj, An Algebraic Geometry Algorithm for Scheduling in the Presence of Setups and Correlated Demands, Mathematical Programming 69 (1995) 369-401.

[35] B. Vizvári, Beiträge zum Frobenius Problem, D.Sc.Nat. Dissertation, Technische Hohschule Carl Schorlemmer, Leuna-Merseburg, Germany, 1987. 